\title{
The Cosmic Microwave Background and Scalar-Tensor Theories of Gravity
}

\author{
C. A. Clarkson, ${ }^{*}$ A. A. Coley ${ }^{\dagger}$ and E. S. D. O’Neill ${ }^{\ddagger}$ \\ Department of Mathematics and Statistics, Dalhousie University, Halifax, Nova Scotia, Canada, B3H 3 J5.
}

(October 31, 2018)

\begin{abstract}
We show that if all observers see an isotropic cosmic microwave background in an expanding geodesic perfect fluid spacetime within a scalar-tensor theory of gravity, then that spacetime must be isotropic and spatially homogeneous. This result generalizes the Ehlers-Geren-Sachs Theorem of General Relativity, and serves to underpin the important result that any evolving cosmological model in a scalar-tensor theory that is compatible with observations must be almost FriedmannLemaître-Robertson-Walker.
\end{abstract}

\section{INTRODUCTION}

The extremely high isotropy of the cosmic microwave background (CMB), which is isotropic to one part in $10^{5}$, provides the strongest evidence that the Universe is isotropic about us. Unfortunately, the spatial homogeneity of the Universe cannot then be inferred (from our observations of the CMB) without the Copernican principle (or some equivalent). However, if we assume the Copernican principle, in that we assume that all observers in the Universe see the CMB to be as isotropic as we see it, we can then ask: can we infer spatial homogeneity on the basis of the CMB observations alone? The first attempt to answer this question within general relativity (GR) resulted in a theorem by Ehlers, Geren and Sachs (hereafter, EGS; [1]) which states that if all observers in a dust universe see an isotropic radiation field then that spacetime is spatially homogeneous and isotropic (Friedmann-Lemaitre-Robertson-Walker, FLRW), in which the isotropic radiation field may be implicitly identified with the CMB.

The EGS theorem can be generalised trivially to the case of a geodesic and barotropic perfect fluid [2]. However, as has been emphasised [3,4] the resulting spacetime will be FLRW only if the matter content is of perfect fluid form and the observers are geodesic and irrotational. Indeed, the EGS theorem has recently been investigated in inhomogeneous universe models with non-geodesic observers [3]; inhomogeneous spacetimes have been found which also allow every observer to see an isotropic CMB. It has also been shown that a significant subset of these models are consistent with other observational constraints, regardless of observer position [5]. This means that these models are consistent with observations even when the Copernican principle is taken into account - and yet the models are significantly inhomogeneous.

The purpose of this paper is to investigate cosmological models which allow an isotropic radiation field in more general theories of gravity (than GR). In particular, we shall study scalar-tensor theories of gravity [6.7], in which gravity is mediated by a long-range scalar field in addition to the usual tensor fields present in Einstein's theory. Such theories of gravitation, and especially the simple Brans-Dicke theory of gravity (BDT; [7] ), are perhaps the most natural alternatives to GR. Observational limits [8] on the present value of (the Brans-Dicke parameter) $\omega_{0}$ need not constrain the value of $\omega$ at early times in more general scalar-tensor theories (than BDT) with a variable $\omega(\phi)$ [10]. Hence, more recently there has been greater focus on the early Universe predictions of scalar-tensor theories of gravity. Scalar-tensor theories with a 'free' scalar field are perhaps not well motivated since, often, quantum corrections produce interactions resulting in a non-trivial potential $U(\phi)$.

Scalar-tensor gravity theory is currently of particular interest since such theories occur as the low-energy limit in supergravity theories from string theory [1] and other higher-dimensional gravity theories [12]. Perhaps cosmology is the ideal setting in which to study possible stringy effects. However, lacking a full non-perturbative formulation which allows a description of the early Universe close to the Planck time, it is necessary to study classical cosmology prior to the GUT epoch by utilizing the low-energy effective action induced by string theory. To lowest order in the inverse string tension the tree-level effective action in four-dimensions for the massless fields includes the nonminimally coupled graviton, the scalar dilaton and an antisymmetric rank-two tensor resulting in a four-dimensional

\footnotetext{
*Email: clarkson@mathstat.dal.ca

${ }^{\dagger}$ Email: aac@mathstat.dal.ca

${ }^{\ddagger}$ Email: oneill@mathstat.dal.ca
} 
scalar-tensor theory of gravity, thereby generalizing GR by including other massless fields. As a result, BDT includes the dilaton-graviton sector of the string effective action as a special case $(\omega=-1)$ [11].

The general form of the extended gravitational action in scalar-tensor theories is

$$
S=\frac{1}{16 \pi} \int d^{4} x \sqrt{-g}\left[\phi R-\frac{\omega(\phi)}{\phi}(\nabla \phi)^{2}-U(\phi)+2 \mathcal{L}_{\text {matter }}\right],
$$

where $\mathcal{L}_{\text {matter }}$ is the Lagrangian for the matter fields, which we shall assume here corresponds to a comoving perfect fluid. We use units in which $c=G=1$. BDT corresponds to the particular choice of $U=0$ and $\omega=$ const. (strictly speaking, $U(\phi)$ must be set to zero in order to ensure a Newtonian weak field limit). The post-Newtonian parameters of general relativity are also recovered in the limit that $\omega \rightarrow \infty$ and $\left(\phi / \omega^{3}\right)(d \omega / d \phi) \rightarrow 0$ [8]. The case where $U(\phi)$ is non-zero but $\omega=0$ is equivalent to higher-order gravity theories [13] with Yukawa type corrections to the Newtonian potential.

The field equations, obtained by varying the action (1) with respect to the metric and the field $\phi$, are

$$
\begin{gathered}
G_{a b}=\frac{8 \pi}{\phi} T_{a b}+\frac{\omega}{\phi^{2}}\left(\nabla_{a} \phi \nabla_{b} \phi-\frac{1}{2} g_{a b} \nabla_{c} \phi \nabla^{c} \phi\right)+\phi^{-1}\left(\nabla_{a} \nabla_{b} \phi-g_{a b} \nabla_{c} \nabla^{c} \phi\right)-\frac{1}{2} g_{a b} U(\phi) . \\
(3+2 \omega) \nabla_{a} \nabla^{a} \phi=8 \pi T-\nabla_{a} \omega \nabla^{a} \phi+\frac{d U}{d \phi}
\end{gathered}
$$

where the energy-momentum tensor $T^{a b}=2\left(\delta \mathcal{L}_{\text {matter }} / \delta g_{a b}\right)$, will take the form of a perfect fluid [see (8), below]. It is known that scalar-tensor theories can be rewritten in the conformally related 'Einstein' frame. However, we shall not do this here and we shall work in the so-called 'Jordan' frame. In the scalar-tensor gravity theories the principle of equivalence is guaranteed by requiring that all matter fields are minimally coupled to the metric $g_{a b}$. Thus energy-momentum is conserved:

$$
\nabla^{a} T_{a b}=0 .
$$

In the following section we prove that in scalar-tensor theories of gravity, the only geodesic perfect fluid spacetimes admitting an isotropic radiation field are non-expanding or have a Robertson-Walker (RW) geometry. The procedure is to first show that the fluid congruence must be irrotational, from which it follows from the field equations that the effective energy flux of the Einstein tensor (discussed below) must vanish. Further study of the evolution of the effective anisotropic pressure tensor reveals that it too must vanish or the spacetime must be stationary. Thus we arrive at the above conclusion.

\section{SPACETIMES ADMITTING AN ISOTROPIC RADIATION FIELD}

\section{A. Isotropic Radiation and Kinematics}

In GR, assuming that all observers on some congruence $u^{a}$ see an exactly isotropic radiation field, then this velocity field has two important properties:

$$
\begin{aligned}
\nabla_{[a}\left(\dot{u}_{b]}-\frac{1}{3} \theta u_{b]}\right) & =0, \\
\sigma_{a b} & =0,
\end{aligned}
$$

where $\theta, \dot{u}^{a}$ and $\sigma_{a b}$ are, respectively, the expansion, acceleration and the shear for the timelike congruence $u^{a}$ (we follow the notation of 14] throughout). This 'isotropic radiation field theorem' may be derived from the EinsteinBoltzmann equations for photons in a curved spacetime [14,1,2]. As the theorem does not involve Einstein's field equations, it may be carried over to scalar-tensor theories without change. The first part of condition (5) allows us to define $\nabla_{a} Q \equiv \dot{u}_{a}-\frac{1}{3} \theta u_{a}$, for some scalar function $Q$ (proportional to the logarithm of the energy density of the radiation), so we see that the first condition (5) is equivalent to

$$
\dot{u}_{a}=\widetilde{\nabla}_{a} Q, \quad \theta=3 \dot{Q},
$$

and it follows that spacetimes admitting an isotropic radiation field are conformally stationary (as may be seen by considering a conformal transformation of the congruence, with $e^{Q}$ as the conformal factor multiplying a stationary metric, with the velocity fields of the two spacetimes parallel - see [9]). 
We shall restrict our attention to the case in which the physical fluid is geodesic (i.e., acceleration-free), which then implies that the rotation of $u^{a}, \omega_{a b}$, must also vanish: following the argument in [3], from (6) we can write

$$
\dot{u}_{a}=\widetilde{\nabla}_{a} Q=0
$$

so that

$$
0=\widetilde{\nabla}_{[a} \dot{u}_{b]}=\widetilde{\nabla}_{[a} \widetilde{\nabla}_{b]} Q=\omega_{b a} \dot{Q}=\frac{1}{3} \omega_{a b} \theta
$$

and we see that $\omega_{a b}=0$ when $\theta \neq 0$. In this case there exist comoving coordinates in which the metric takes the form given in 15 .

\section{B. The Matter and the Einstein Tensor}

We are assuming the matter may be described by a perfect fluid, so the energy momentum tensor has the form:

$$
T_{a b}=\mu u_{a} u_{b}+\frac{1}{3} \mu h_{a b}+\rho u_{a} u_{b}+p h_{a b},
$$

i.e., the matter is a mixture of radiation (which may, without loss of generality, be considered as a test field, thereby contributing nothing to the gravitational field) and some other type of perfect fluid satisfying $\widetilde{\nabla}_{a} p=0$ [which, via (4), ensures the observers are geodesic]. The Einstein tensor must then satisfy (2), with the matter given by (8).

In general, the Einstein tensor can formally be decomposed with respect to a timelike vector field $u^{a}$ according to

$$
G_{a b} \equiv \mu^{\text {eff }} u_{a} u_{b}+p^{\text {eff }} h_{a b}+q_{a}^{\text {eff }} u_{b}+q_{b}^{\text {eff }} u_{a}+\pi_{a b}^{\text {eff }}=T_{a b}^{\text {eff }}
$$

where

$$
q_{a}^{\mathrm{eff}} u^{a}=0, \quad h^{a b} \pi_{a b}^{\mathrm{eff}}=0, \quad \pi_{a b}^{\mathrm{eff}} u^{b}=0 .
$$

$T_{a b}^{\mathrm{eff}}$ is now an effective energy-momentum tensor, which is not related to the physical fluid given by (8) other than by (2). In this formal decomposition $\mu^{\text {eff }}, p^{\text {eff }}, q_{a}^{\text {eff }}$ and $\pi_{a b}^{\text {eff }}$ are given by

$$
\begin{aligned}
\mu^{\text {eff }} & =G_{a b} u^{a} u^{b}, \\
p^{\text {eff }} & =\frac{1}{3} h^{a b} G_{a b}, \\
q_{a}^{\text {eff }} & =-h_{a}{ }^{c} G_{c d} u^{d}, \\
\pi_{a b}^{\text {eff }} & =h_{a}{ }^{c} h_{b}{ }^{d} G_{c d}-\frac{1}{3}\left(h^{c d} G_{c d}\right) h_{a b}=G_{\langle a b\rangle} .
\end{aligned}
$$

(Angled brackets denote the projected, symmetric, and trace-free part of tensors, as defined by (14); see 14].) For a fluid with four-velocity $u^{a}$, these quantities denote, via the field equations, the energy density, isotropic pressure, energy flux and anisotropic pressure, respectively, as measured by an observer comoving with the fluid. Using this decomposition, we may decompose (2) into the effective energy density and pressure, energy flux and anisotropic pressure. Using this decomposition of (2), given by (9) - (14), we may now use the field equations of GR in the $1+3$ covariant formalism [14], but replacing the relevant quantities with their effective counterparts $\left(\right.$ i.e., so that $\mu \rightarrow \mu{ }^{\text {eff }}$, etc.).

When $\dot{u}_{a}$ and $\omega_{a b}$ are zero, (5) becomes

$$
\nabla_{[a}\left(\theta u_{b]}\right)=u_{[b} \nabla_{a]} \theta=0
$$

which implies (since $\left.\nabla_{a} \theta=\widetilde{\nabla}_{a} \theta-\dot{\theta} u_{a}\right)$ that

$$
\widetilde{\nabla}_{a} \theta=0
$$

(i.e., the expansion is spatially homogeneous). From the constraint equation relating the divergence of the shear to other kinematical quantities (Eq., (32) in [14) we see that any energy flux component of the Einstein tensor (or, equivalently, of $T_{a b}^{\text {eff }}$ ) with respect to the perfect fluid velocity field must vanish:

$$
q_{a}^{\text {eff }}=\frac{2}{3} \widetilde{\nabla}_{a} \theta=0
$$


However, from (2), and using (13), we see that the effective energy flux of the Einstein tensor is

$$
q_{a}^{\text {eff }}=-G_{\langle a\rangle b} u^{b}=\phi^{-1}\left(\widetilde{\nabla}_{a} \phi\left[\frac{1}{3} \theta-\omega(\ln \phi)^{\cdot}\right]-\widetilde{\nabla}_{a} \dot{\phi}\right)=0 .
$$

Note that we now have a spacetime whose Einstein tensor may be written, using (11) - (14), as a fluid with zero heat flux with respect to a geodesic, shearfree and irrotational congruence; hence, the effective anisotropic pressure vanishing is a necessary and sufficient condition for the spacetime to be RW [14, 15], and we now show that this must be the case.

\section{Proof of the main result}

From $(17)$ we see that these spacetimes must satisfy

$$
\left(\widetilde{\nabla}_{a} \phi\right)^{\cdot}=-\omega(\ln \phi)^{\cdot} \widetilde{\nabla}_{a} \phi
$$

in order to admit an isotropic radiation field, where we have used the identity

$$
\widetilde{\nabla}_{a} \dot{\xi}=h_{a}^{b}\left(\widetilde{\nabla}_{b} \xi\right)^{\cdot}-\dot{\xi} \dot{u}_{a}+\frac{1}{3} \theta \widetilde{\nabla}_{a} \xi+\sigma_{a}^{b} \widetilde{\nabla}_{b} \xi+\eta_{a b c} \omega^{b} \widetilde{\nabla}^{c} \xi,
$$

to interchange the order of 'time' and 'space' derivatives of scalars. Equation (18) may be integrated to give

$$
\widetilde{\nabla}_{a} \phi=\Phi(\phi) F_{a},
$$

where

$$
\Phi(\phi)=\exp \left(-\int \omega \frac{d \phi}{\phi}\right),
$$

and $F^{a}$ is an arbitrary spatial vector field such that $\dot{F}^{a}=0=u^{a} F_{a}$. Now that $\phi$ must satisfy (20), the total effective anisotropic pressure tensor from (2) and (14),

$$
\pi_{a b}^{\text {eff }}=\frac{1}{\phi} \widetilde{\nabla}_{\langle a} \widetilde{\nabla}_{b\rangle} \phi+\frac{\omega}{\phi^{2}} \widetilde{\nabla}_{\langle a} \phi \widetilde{\nabla}_{b\rangle} \phi
$$

becomes

$$
\pi_{a b}^{\text {eff }}=\frac{\Phi}{\phi} \widetilde{\nabla}_{\langle a} F_{b\rangle}
$$

In order to proceed, let us now use the field equations to find further restrictions on $\phi$. From the evolution equation for the electric Weyl tensor, and the shear evolution equation, we obtain

$$
E_{a b}=\frac{1}{2} \pi_{a b}^{\text {eff }}
$$

so therefore

$$
\dot{\pi}_{\langle a b\rangle}^{\mathrm{eff}}=-\frac{2}{3} \theta \pi_{a b}^{\mathrm{eff}} .
$$

However, from (23) we may calculate the time derivative of $\pi_{a b}$,

$$
\dot{\pi}_{\langle a b\rangle}^{\text {eff }}=\frac{\Phi}{\phi}\left\{-\frac{\dot{\phi}}{\phi}(1+\omega) \widetilde{\nabla}_{\langle a} F_{b\rangle}+\left(\widetilde{\nabla}_{\langle a} F_{b\rangle}\right)\right\} .
$$

Using the Ricci identities, and the fact that $R_{a\langle b c\rangle d} u^{a} F^{d}=0$ in our case, we find that

$$
\left(\widetilde{\nabla}_{\langle a} F_{b\rangle}\right)=-\frac{1}{3} \theta \widetilde{\nabla}_{\langle a} F_{b\rangle},
$$

and so, using (25.26), we find that 


$$
\left[\frac{\dot{\phi}}{\phi}(1+\omega)-\frac{1}{3} \theta\right] \widetilde{\nabla}_{\langle a} F_{b\rangle}=0 .
$$

Hence we have that $\widetilde{\nabla}_{\langle a} F_{b\rangle}=0$, which implies that $\pi_{a b}^{\text {eff }}=0$ and consequently the spacetime is RW, or

$$
\theta=3 \frac{\dot{\phi}}{\phi}(1+\omega)
$$

If we now take the spatial gradient of this expression we obtain, using 17,

$$
0=\widetilde{\nabla}_{a} \theta=3 \omega^{\prime} \frac{\dot{\phi}}{\phi} \widetilde{\nabla}_{a} \phi
$$

where $\omega^{\prime} \equiv \frac{d \omega}{d \phi}$. Now, if $\widetilde{\nabla}_{a} \phi=0$, then $\pi_{a b}^{\text {eff }}=0$, and again the spacetime is RW. If $\dot{\phi}=0$ then the expansion is zero, and the spacetime is stationary [recall that if $\theta=0$ then the rotation need not vanish (7)]. The only other possibility remaining is that $\omega$ is constant and that $\widetilde{\nabla}_{a} \frac{\dot{\phi}}{\phi}=0$. Integrating this expression we find that $\phi$ is a separable function (in time and space in the coordinates of 15]), whence on inserting this into equation (20) we find that for $\omega=$ constant $\neq-1$ this leads to $\widetilde{\nabla}_{a} \phi=0$ or $\dot{\phi}=0$; i.e., the spacetime must again be either RW or stationary [and in the case $\omega=-1,(29)$ ensures the spacetime is non-expanding].

\section{Discussion}

Consequently we have shown that in all cases the spacetime must be non-expanding or $R W$. In particular, all evolving spacetimes must necessarily have an isotropic and spatially homogeneous geometry. This implies that the total energy-momentum tensor must be of perfect fluid form whose components depend on cosmic time only. This does not, however, imply that each of the separate components of the energy-momentum tensor, such as for example the scalar field $\phi$, need individually be functions of time alone [16]. We note that the preceding calculation did lead to additional restrictions on $\phi$, namely equations (18) and (20) must be satisfied and $\widetilde{\nabla}_{\langle a} F_{b\rangle}=0$. However, if we take the field equations (2) and 'contract' them using $u^{a} u^{b}$ and $g^{a b}$, respectively, using the Brans-Dicke field equation (3) to substitute for $\nabla_{a} \nabla^{a} \phi$, we obtain two expressions which can be written, using the equations above, as a sum of terms each of which has a particular (separable) dependence on time, space or a functional dependence on $\phi$. Setting these two expressions equal to zero, it is then straightforward to show that these two equations can only be satisfied in general for $\phi=\phi(t)$. Hence it follows that all physical quantities can only depend on time alone.

\section{CONCLUSIONS}

We have discussed some of the consequences of the isotropy of the CMB in universe models in scalar-tensor theories of gravity. We have shown that, if we assume geodesic motion and a perfect fluid matter source, any expanding spacetime must have RW geometry, which is also the situation in GR. As is the case in GR, we would expect that this result will have a perturbed equivalent, in that the near isotropy of the CMB will imply a nearly homogeneous and isotropic cosmological model in these alternative theories of gravity [17]. Thus we conclude that if we wish to consider cosmological implications of scalar tensor theories of gravity, $\mathrm{CMB}$ observations imply that the canonical 'almost' FLRW models will be the correct models to use, provided that other observations fulfill the assumptions used here.

\section{ACKNOWLEDGMENTS}

We would like to thank Roy Maartens for useful comments. This work was funded in part by NSERC of Canada. 
[1] J. Ehlers, P. Geren, and R. K. Sachs, J. Math. Phys. 91344 (1964).

[2] G. F. R. Ellis, D. R. Matravers, and R. Treciokas, Ann. Phys. 150, 455 (1983); 150, 487 (1983).

[3] C. A. Clarkson and R. K. Barrett, Class. Quantum Grav. 16, 3781 (1999).

[4] J. J. Ferrando, J. A. Morales, and M. Portilla, Phys. Rev. D 46(2), 578 (1992).

[5] R. K. Barrett and C. A. Clarkson, Class. Quantum Grav. 175047 (2000) astro-ph/9911235, C. A. Clarkson, Ph. D. Thesis, University of Glasgow (1999) astro-ph/0008089.

[6] P. Jordan, Z. Phys. 157112 (1959); P. G. Bergmann, Int. J. Theor. Phys. 125 (1968); R. V. Wagoner, Phys. Rev. D1 3209 (1970); K. Nordtvedt, Astrophys. J. 161, 1059 (1970).

[7] C. Brans and R. H. Dicke, Phys. Rev. 124, 925 (1961).

[8] C.M. Will, Theory and Experiment in Gravitational Physics (Cambridge University Press, Cambridge) (1993).

[9] D. Kramer et. al. Exact Solutions of Einstein's Field Equations, Cambridge University Press. (1980).

[10] J.D. Barrow and P. Parsons, Phys. Rev. D. 55, 1906 (1997).

[11] M.B. Green, J.H. Schwarz and E. Witten, Superstring Theory (Cambridge: Cambridge University Press) (1988).

[12] T. Applequist, A. Chodos and P.G.O. Freund, Modern Kaluza-Klein Theories (Redwood City: Addison-Wesley) (1987).

[13] D. Wands, Class. Quantum Grav. 7269 (1994).

[14] G. F. R. Ellis and H. van Elst, in M. Lachieze-Rey (ed.), Theoretical and Observational Cosmology, NATO Science Series, Kluwer Academic Publishers (1998) gr-qc/981204004; G F R Ellis General Relativity and Cosmology Proceedings of XLVII Enrico Fermi Summer School, ed. R K Sachs (New York: Academic Press) (1971).

[15] A. A. Coley and D. J. McManus, Class. Quantum. Grav. 111261 (1994).

[16] A. A. Coley and B. O. J. Tupper, 1983, Astrophys. J. 217, 1; ibid., 1986; J. Math. Phys. 27406.

[17] W. R. Stoeger, R. Maartens, and G. F. R. Ellis, Astrophys. J. bf 443, 1 (1995); R. Maartens, G. F. R. Ellis, and W. R. Stoeger, Phys. Rev. D 51, 1525 (1995); 51, 5942 (1995); Astrophys. J., 309, L7 (1996). 